\title{
Amélioration des aliments végétaux par les protéines du lactosérum
}

\author{
par \\ E. RENNER*, Maria C. BARRIO DE BRESSANELLO**, \\ R. BACCHETTA, E. CASTELAO, R. GONZALEZ, \\ H. SANCHEZ***
}

\section{Résu mé}

Le but de l'étude était d'examiner dans quelle mesure on peut utiliser des concentrés de protéines lactosériques, obtenus par ultrafiltration de lactosérum, pour enrichir en protéines le pain et les nouilles. A cet effet, on a mélangé à la farine 3 portions différentes de concentrés de protéines lactosériques à taux protéiques variables $(35,45$ et $60 \%)$ et à degrés de dénaturation différents (faible, moyen, fort).

Plus la quantité ajoutée était grande, plus le volume spécifique du pain enrichi diminuait par rapport à l'échantillon de contrôle. La même tendance a été observée lors de l'appréciation sensorielle basée sur une échelle de 100 points. Des résultats favorables ont été obtenus après adjonction d'un concentré de protéines lactosériques à degré de dénaturation et à taux protéique moyens. Une déviation sensorielle statistiquement significative par rapport au contrôle n'a été enregistrée que pour les échantillons enrichis de $6 \%$ de concentré protéique. Le goût du pain enrichi n'était cependant pas jugé plus défavorablement que celui de l'échantillon témoin.

Quant à l'enrichissement protéique des nouilles, on a constaté que seule une adjonction trop élevée de concentré de protéines lacto-

\footnotetext{
* Université Justus Liebig, Giessen (Rép. Féd. d'Allemagne).

** INTI/CITIL, Buenos Aires (Argentina).

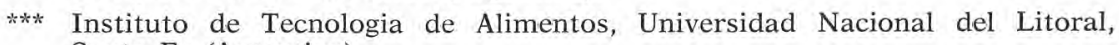
Santa Fe (Argentina).
} 
sériques, aboutissant à une teneur en protéines de $20 \%$ du produit fini, avait une influence défavorable sur sa structure.

Les pertes à la cuisson ne sont pas beaucoup plus importantes pour les produits enrichis en protéines. Pour les nouilles également, ce n'est qu'après une adjonction de quantités supérieures de concentré protéiques qu'on observe des effets sur la qualité gustative, cependant assez faibles.

Grâce à la haute teneur en acides aminés essentiels des protéines lactosériques, la valeur biologique des aliments végétaux enrichis est meilleure que celle des échantillons de contrôle. On peut donc attribuer une grande importance au pain et aux nouilles enrichis en concentrés de protéines lactosériques, en particulier pour l'alimentation dans les pays en développement.

\section{S u m m a ry}

\section{PROTEIN FORTIFICATION OF VEGETABLE FOODS WITH WHEY PROTEIN CONCENTRATE}

In the frame of a joint project it was investigated, whether whey protein concentrate (WPC), which results from the ultrafiltration of whey, can be used for the protein enrichment of different vegetable foods like bread and noodles. Therefore whey protein concentrates with different protein content $(30,45$ and $60 \%)$ and with a varying denaturation degree (low - medium - high) were mixed were mixed with flour in 3 different proportions.

By adding whey protein concentrate the specific volume of bread is reduced compared with the control samples. The same tendency can be observed as to the sensoric evaluation using a point-scheme. Fairly good results are obtained by using a concentrate with a medium denaturation degree and a medium protein content. By the sensoric "tetrade test" significant differences only could be observed at an amount of $6 \%$ added WPC, but the panelists certified no unfavorable taste quality to the protein fortified bread.

As to the protein fortification of noodles it was recognized, that only a too high addition of WPC, which gives a protein content of $20 \%$, has an unfavorable effect on the structure. The cooking losses are increasing in the fortified product only in a small degree. Also in this case merely a high addition of WPC has a relatively little effect on the taste quality.

Because of the high content of essential amino acids in whey protein the biological value of protein-fortified vegetable foods is increased compared with the control samples. Therefore bread and noodles, which were enriched by WPC, have a specific importance for the human nutrition in developing countries. 


\section{INTRODUCTION}

Lors de la fabrication de fromages, quelque 75 millions de tonnes de lactosérum sont produits chaque année dans le monde entier. Cela équivaut à environ $450000 \mathrm{t}$ de protéines du lactosérum, qui présentent une valeur biologique très élevée. Autrefois, cette masse de protéines a souvent été traitée comme déchet. En partie, elle a été utilisée dans l'alimentation des animaux. Actuellement on s'efforce de profiter des protéines lactosériques si précieuses du point de vue nutritionnel en les intégrant dans l'alimentation humaine.

Dans le cadre d'un projet de recherches entrepris en collaboration avec des instituts argentins, nous avons étudié dans quelle mesure on peut utiliser des concentrés de protéines lactosériques, obtenus par ultrafiltration du lactosérum, pour enrichir des aliments végétaux. Nous avons déjà réalisé des essais avec du pain et des nouilles (spaghetti) ; une autre série expérimentale sera consacrée à l'enrichissement protéique de biscuits secs.

Le but principal de ces essais est d'examiner si, par l'adjonction d'un concentré de protéines lactosériques, on peut maintenir ou améliorer la qualité de ces aliments tout en augmentant leur valeur nutritive.

\section{EXECUTION DES ESSAIS}

Les essais portaient sur différents types de concentrés de protéinès lactosériques :

- concentrés à 3 taux de protéines différents, à savoir 35,45 et $60 \%$ (tab. 1) ;

- concentrés à différents degrés de dénaturation. L'adjoncttion de concentrés protéiques du lactosérum à des aliments solides requiert un certain degré de dénaturation des protéines. Par contre, si les protéines lactosériques ont été dénaturées entièrement par une précipitation, elles influencent très défavorablement la qualité des aliments auxquels elles sont ajoutées ;

- les protéines lactosériques ayant été ajoutées aux aliments à 3 degrés de concentration différents, de manière que 9 variantes d'essai au total ont été réalisées.

\section{PAIN ENRICHI EN PROTEINES}

\section{Influence sur la cuisson}

Dans les essais destinés à examiner l'enrichissement protéique du pain, le concentré de protéines lactosériques séché a été ajouté à la farine à raison de 2,4 et $6 \%$. 
TABLEAU 1 - TABLE 1

Composition des concentrés de protéines lactosériques

Composition of whey protein concentrates

\begin{tabular}{l|c|c|c}
\hline \multirow{2}{*}{ Elément } & \multicolumn{2}{|c}{ Teneur en \% de la matière sèche } \\
\cline { 2 - 4 } & PF & PM & PE \\
\hline Protéine & 35 & 45 & 60 \\
Matière grasse & 3.5 & 4.5 & 6.0 \\
Eléments minéraux & 6 & 5 & 4 \\
Lactose & 47 & 38 & 23 \\
\hline
\end{tabular}

Teneur de protéines $: \mathrm{PF}=$ faible $; \mathrm{PM}=$ moyenne $; \mathrm{PE}=$ élevée.

En examinant les effets de cet enrichissement sur la cuisson, nous avons constaté que l'absorption de l'eau diminue avec l'augmentation du degré de substitution. Cela peut s'expliquer par une influence éventuelle des protéines lactosériques sur les propriétés du gluten. Comme il ressort des alvéogrammes, les protéines lactosériques d'un degré de dénaturation moyen ont un comportement semblable à celui des protéines des céréales. En général, les protéines lactosériques dont le degré de dénaturation est élevé donnent une pâte plus épaisse, alors que la pâte additionnée de protéines peu dénaturées est plus élastique.

\section{Influence sur les propriétés du pain}

L'adjonction de concentrés de protéines lactosériques (CPL) réduit quelque peu le volume spécifique par rapport aux échantillons de contrôle (fig. 1). Les meilleurs résultats sont obtenus quand on utilise un concentré à degré de dénaturation et à taux de protéines moyens. Quand on se sert du concentré à degré de dénaturation élevé, le volume spécifique du pain diminue avec l'augmentation de la quantité d'adjonction.

Les pains d'essai ont été soumis à une évaluation sensorielle, basée sur une échelle de 100 points, effectuée par un examinateur expérimenté. Les propriétés suivantes ont été appréciées : volume, croûte, texture interne et structure, couleur à l'intérieur du pain, odeur et goût. Il convient de mentionner que même l'échantillon de contrôle n'a pas obtenu le maximum de 100 points lors de cette appréciation. Dans les pains enrichis en protéines, on note la même 


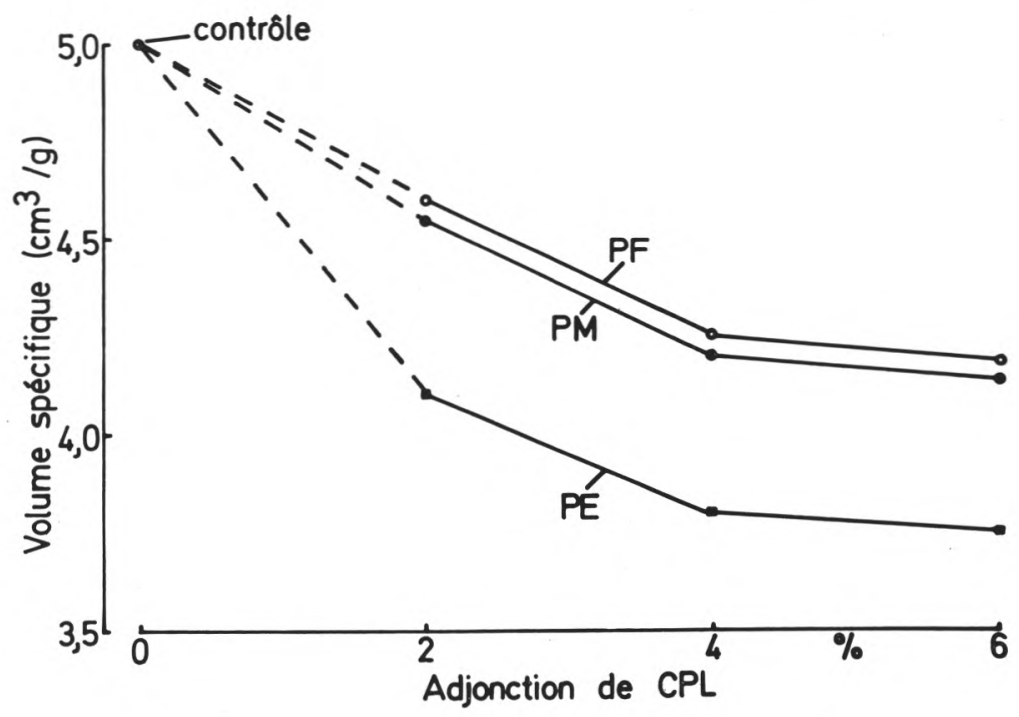

fig. 1

Influence de l'adjonction de concentrés de protéines lactosériques sur le volume spécifique du pain.

Influence of adding whey protein concentrates on the specific volume of bread.

\section{TABLEAU 2 - TABLE 2}

Résultats de "tetrade test " sur la différence organoleptique entre le pain avec CPL ajouté et le contrôle

Results of the tetrade test on the organoleptic difference between protein-fortified bread and the control

\begin{tabular}{c|c|c|c}
\hline \multirow{2}{*}{$\begin{array}{c}\text { Adjonction de CPL } \\
\text { en \% }\end{array}$} & \multicolumn{3}{|c}{ Significance de différence } \\
\cline { 2 - 4 } & PF & PM & PE \\
\hline 2 & + & + & ++ \\
4 & ++ & ++ & +++ \\
6 & +++ & +++ & +++
\end{tabular}

Significance statistique : $+=$ faible $;++=$ moyenne $;+++=$ haute. 
tendance constatée pour le volume spécifique : les résultats de l'appréciation sensorielle sont moins favorables lorsqu'on ajoute une grande quantité d'un concentré à degré de dénaturation élevé (fig. 2). En revanche, les résultats d'appréciation sont positifs quand le concentré présente un degré de dénaturation et un taux de protéines moyens.

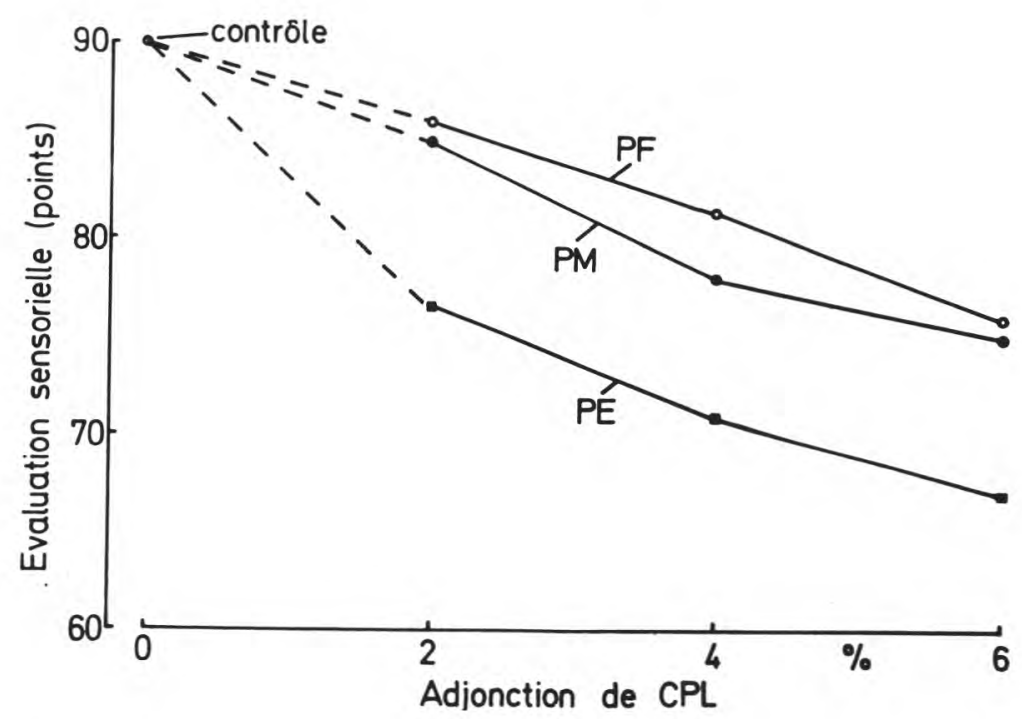

fig. 2

Influence de l'adjonction de concentrés de protéines lactosériques sur l'élévation sensorielle.

Influence of adding whey protein concentrates on the sensoric quality of bread.

Une autre appréciation a été effectuée à l'aide du "tetrade test " par un panel de 11 personnes pour examiner si des différences significatives existent entre le goût des pains d'essais et celui des contrôles.

Principalement une adjonction de $6 \%$ de concentré de protéines lactosériques a donné un goût significativement différent. Le goût des pains enrichis n'a cependant pas été jugé plus défavorablement que celui des échantillons de contrôle.

\section{Valeur nutritive}

On augmente le taux des protéines du pain de 12,8 à 15,9\%, par exemple, en ajoutant, à raison de $6 \%$, un concentré à teneur protéique élevée. Comme les protéines lactosériques, grâce à leur haute 
concentration en acides aminés essentiels, ont une valeur biologique supérieure, il est évident que celle du pain enrichi est aussi considérablement améliorée. Cela a été confirmé par plusieurs essais. L'adjonction de $6 \%$ d'un concentré à haute teneur protéique accroît la concentration de presque tous les acides aminés essentiels du pain, comme le montre la figure 3 . La composition en acides aminés de ce pain enrichi en protéines correspond largement à celle de la protéine de référence, fixée par la FAO/WHO pour l'alimentation humaine. Ce type de pain enrichi en protéines lactosériques pourrait sans doute jouer un rôle très important pour l'alimentation des pays en voie de développement.

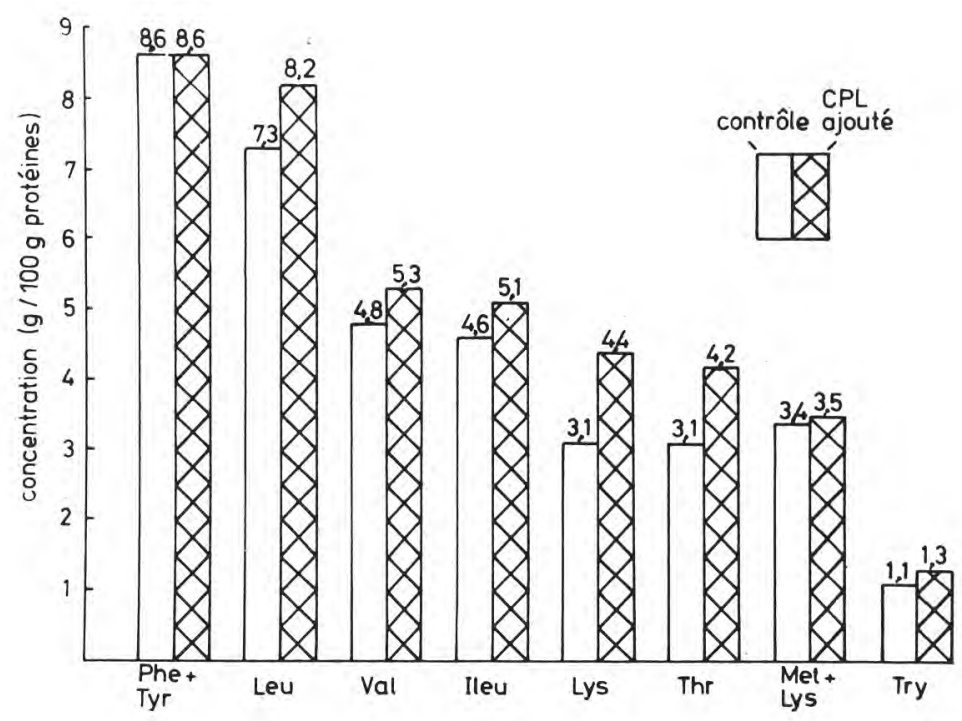

fig. 3

Concentration des acides aminés essentiels du pain. Concentration of essential amino acids in bread.

\section{NOUILLES ENRICHIES EN PROTEINES}

\section{Influence sur la fabrication}

Pour les essais portant sur l'enrichissement en protéines de nouilles, la quantité d'adjonction des concentrés a été fixée en fonction des teneurs finales, soit 16,18 ou $20 \%$ de protéines dans la matière sèche du produit fini. Les échantillons témoins contenaient $12 \%$ de protéines. 
Avec l'abaissement du degré de dénaturation et l'augmentation de la quantité ajoutée de concentrés de protéines lactosériques, l'absorption de l'eau du mélange diminue. Cette absorption est cependant nécessaire au conditionnement avant l'extrusion des nouilles. Les produits additionnés d'un concentré de protéines fortement dénaturées ont été jugés de la manière la plus favorable. Mélangées avec la farine, les protéines lactosériques à degré de dénaturation moyen ou bas donnèrent une pâte un peu trop épaisse.

\section{Influence sur les propriétés des nouilles}

On a constaté que la structure des nouilles finies ayant une teneur en protéines de $16 \%$ est semblable à celle des échantillons de contrôle. Les produits d'un taux protéique de $18 \%$ étaient peu différents des échantillons de contrôle, alors que les nouilles contenant $20 \%$ de protéines étaient cassantes. En pratique, une adjonction aussi forte n'est donc guère recommandable.

Les pertes à la cuisson s'élevaient à $4 \%$ pour les contrôles et à 5 à $8 \%$ (ce qui n'est pas beaucoup plus) pour les nouilles enrichies d'un concentré à taux protéique moyen ou élevé. Par contre, l'addition d'un concentré à taux protéique bas entraînait des pertes assez importantes après un temps de cuisson de $10 \mathrm{~min}$.

La texture dite «al dente» a pu être atteinte pour les nouilles de toutes les variantes ; pour les nouilles enrichies d'une grande quantité de concentré protéique, il fallait uniquement prolonger le temps de cuisson.

Pour l'appréciation sensorielle de ce produit, on avait de nouveau recours au « tetrade test », effectué par un panel de 11 examinateurs. Quant aux nouilles enrichies contenant 18 et $20 \%$ de protéines, on enregistra, par rapport aux contrôles, une déviation du goût statistiquement significative que les examinateurs qualifièrent cependant d'insignifiante à moyenne. Il en ressort qu'une adjonction supérieure de concentré de protéines lactosériques influence le goût des nouilles. Dans l'ensemble, cet effet est relativement faible de façon que le consommateur ne remarque guère les différences gustatives, surtout quand les spaghettis ont été salés lors de la cuisson.

\section{Valeur nutritive}

$\mathrm{Du}$ point de vue nutritionnel, il est souhaitable de fabriquer des nouilles contenant environ $20 \%$ de protéines en ajoutant un concentré de protéines lactosériques. Cela n'est possible qu'en utilisant un concentré à teneur protéique élevée.

Les essais sur la valeur biologique des nouilles enrichies en protéines ne sont pas encore tous achevés. Toutefois, il est permis de penser que la valeur biologique des nouilles peut être améliorée plus nettement encore que celle du pain puisqu'elles tolèrent de plus 
grandes quantités de protéines lactosériques. Cela permettrait d'assurer une alimentation plus complète et précieuse pour les pays de forte consommation de spaghettis.

\section{v. CONCLUSION}

Dans l'ensemble, les essais ont montré qu'une adjonction de concentrés de protéines lactosériques au pain et aux nouilles contribue considérablement à améliorer la valeur nutritive de ces aliments végétaux sans en diminuer essentiellement les qualités organoleptiques. Il est toutefois important de choisir la quantité d'adjonction et le degré de dénaturation de façon à créer des conditions optimales dans le produit fini. 